\title{
Estrategia de inclusión de la historia de la ciencia y la experimentación para construir en e aula el conocimiento científico escolar relacionado con la electrización
}

\begin{abstract}
Strategy of inclusion of the science history and experimentation to construct in the classroom the scholastic scientific knowledge related to electrification
\end{abstract}

Liliana Sandoval Manzano

Instituto de Educación y Pedagogía, Universidad del Valle. lili8san@hotmail.com

\section{Resumen}

En la presente investigación se plantea una propuesta de secuenciación de actividades, mediante el uso de la historia de la ciencia para la enseñanza-aprendizaje-evaluación del concepto de electrización como un fenómeno de estudio fundamental de la física, tomando como punto de partida la vivencia de Tales de Mileto, la cual pretende contribuir en la solución al problema que se encuentra en la enseñanza de las ciencias, donde primero se presenta la teoría y posteriormente se procede a experimentar para comprobar lo que se ha dicho anteriormente; esto genera en nuestros estudiantes de la básica secundaria el no desarrollo de competencias científicas y el no lograr construir significativamente conceptos relacionados con la física.

Dicha propuesta fue construida con un enfoque constructivista basado en el aprendizaje generativo, con miras a fortalecer el proceso de enseñanza aprendizaje y evaluación de la física al igual que promover el desarrollo de competencias científicas, tales como la observación, la interpretación, la argumentación y la propositiva.

\section{Palabras claves}

Actividades experimentales, historia de la ciencia, enseñanza de la física, competencias científicas.

\section{Abstract}

In the present research is presented in a proposal of sequenced activities by using the science history for the educations, learning and evaluation of the electrification concept as a fundamental phenomenon for the physics study, taking like departure point the Mileto's experience, which first the theory appears and later it comes the experiment to verify what it has been said previously; what generates that our students of basic secondary school one do not develop scientific competences and they do not manage to construct concepts related to physics significantly.

This proposal was construted with a constructivist approach based on the generative learning, in order to fortify the teaching and learning process and the physics assessment as well as the promotion of the development of scientific competences like the observation, the interpretation, the argumentation and the propositive competences.

Keyboards

Experimental activities, science history, sequenced activities, scientific of the physics, scientific skills. 


\section{Introducción}

Para logar comprender significativamente el concepto de electrización de los cuerpos, se debe cambiar la forma de enseñanza basada comúnmente en un método tradicional, en el aul se aprende datos sin sentido, de memoria y de forma irreflexiva, dificultad que se refleja en grados posteriores y en la resolución de problemas cotidianos por parte del estudiante. Para superar esta dificultad surge la necesidad de profundizar y reflexionar la forma de enseñar las ciencias particularmente, la física. Desde esta mirada se hace prudente considerar la propuesta constructivista, con el propósito de logar un aprendizaje significativo por parte del estudiante, que le permita aprender ciencias y explicar a través de ella fenómenos de su vida cotidiana, como en este caso sería explicar: ¿Por qué al frotar un cuerpo de determinado material se puede levantar 0 atraer otros más ligeros?. En la enseñanza del conocimiento en torno a la electrización se observa que los estudiantes no logran visualizar que todos los cuerpos poseen carga, y por tanto pueden ser electrizados, sino que ellos limitan esta característica a los cuerpos que comúnmente se nombran (en los libros de texto) en el momento de enseñar dicho concepto por ejemplo: Ámbar, ebonita, vidrio, seda, etc. (Bravo, 2008). A esta dificultad se suma el hecho de que el maestro experimenta muy poco sobre este fenómeno en el aula, y cuando lo hace es para comprobar lo planteado por él o por el libro de texto de forma teórica, dejando de lado la posibilidad de construir la teoría a través de la experimentación, teniendo como base los presupuestos teónicos que tiene el estudiante, De lo anterior, surge la pregunta de investigación: ¿Cómo diseñar una secuencia de actividades experimentales que permita identificar elementos daves históricamente establecidos para la construcción del conocimiento científico escolar, relacionado con el fenómeno de electrización que faculte el desarrollo de competencias científicas en estudiantes de educación básica seaundaria?.

\section{Desarrollo}

Cuando se propone la construcción de la teoría a partir de la experimentación, esta construcción parte de los presupuestos que tiene el estudiante para acercarse a las explicaciones del fenómeno particular, lo que conlleva a tener daridad sobre estos presupuestos. Para el desarrollo de la presente propuesta se toma como hipótesis de investigación, el uso de la historia de la ciencia de tal forma que de visos para la reconstrucción de experimentos históricamente relevantes permitiendo la identificación de elementos daves involucrados en el desarrollo y construcción del conocimiento relacionado con la electrización de los aerpos, como la experiencia inintencionada de Tales de Mileto; haciendo posible la promoción de la construcción del conocimiento científico escolar que faculten el desarrollo de competencias científicas en los estudiantes.

\section{Ideas previas acerca de la electrización}

Al revisar la literatura sobre investigaciones en la enseñanza del conocimiento relacionado con la electricidad estática (Bravo, 2008; Osborne 1991 Ángel y Clavijo 2006) observamos que son muy pocas las que están relacionadas con la indagación de las ideas que presentan los estudiantes sobre el fenómeno de la electrización de los auerpos, por tanto y considerando que son de vital importancia para implementar aualquier estrategia educativa (Driver y Bell, 1986; Osborme 1991; Cubero 1999), se realizó a través de la técnica de auestionario y entrevista (Cubero 1999; Osborne, 1991) una exploración sobre las preconcepciones que poseen nuestros estudiantes con relación a la electrización de los cuerpos pudiéndose establecer que la idea predominantes en los estudiantes en tormo al fenómeno que nos concieme radica en que el estudiante confunde el calentamiento por frotamiento con la causa del fenómeno electrización como se puede observar en la siguiente tabla: 


\begin{tabular}{|c|c|}
\hline PRECONCEPTO & No. DE ESTUDIANTES \\
\hline $\begin{array}{c}\text { Confusión de la acción de las fuerzas } \\
\text { electrostáticas con el calentamiento o calor }\end{array}$ & 9 \\
\hline Por efecto del magnetismo & 1 \\
\hline Confusión de electricidad con energía & $\mathbf{5}$ \\
\hline Relacionadas con los fenómenos eléctricos & 1 \\
\hline
\end{tabular}

Tabla 1. Ideas de los estudiantes en torno al fenómeno de electrización.

De igual forma los estudiantes hablan de la electricidad sea estática o dinámica como sinónimo de energía, sin identificar que la electricidad es una manifestación de la energía. Estas ideas junto con los elementos recogidos en el análisis histónico fueron la clave para la secuenciación de actividades propuesta.

\section{Análisis de la historia: Mileto yGilbert}

Al revisar la historia del fenómeno eléctrico particularmente de la electrización de los cuerpos, vemos que las primeras observaciones se dieron en la antigua Grecia, en el siglo séptimo antes de Cristo. La fortuna permitió que Tales de Mileto, un filósofo que siempre intentaba explicar los fenómenos naturales, realizara las primeras observaciones del fenómeno de atracción del ámbar sobre cuerpos ligeros cuando aquél es frotado previamente, cuando se frotaba el ámbar con piel de animales, éste atraía pelos, plumas y pequeños fragmentos de madera, Tales lo explicó indicando que el ámbar tenía un 'alma' que atraía a los cuerpos; de igual manera explicaba los fenómenos apreciados con la piedra imán. Al analizar esta idea vemos como el hecho de explicar el fenómeno de electrización y el fenómeno magnético por Tales de Mileto concuerda con lo sucedido en el aula de clase, donde el estudiante explica el fenómeno de electrización considerándolo similar al fenómeno magnético, como bien sabemos presentan semejanzas, pero su ocurrencia tiene causas diferentes. La historia evidencia a William Gilbert como el hombre que continuo con las observaciones de Tales, quitando el ingrediente de la casualidad de la vivencia y llevando dicha práctica a la experimentación intencionada, con la cual Gilbert descubrió que muchas otras sustancias tenían la capacidad de atraer objetos ligeros cuando se frotaban y aplicó el término eléctrica para la fuerza ejercida por estas sustancias después de ser frotadas. Otro aporte desde el análisis histórico es el papel que juega la actividad experimental en la construcción del conocimiento relacionado con un fenómeno particular, es decir el observar como los conceptos científicos se construyen históricamente desde la experimentación.

Diseño y secuenciación de actividades.

Para el diseño y secuenciación de las actividades se tuvo en cuenta los siguientes aspectos.

$>$ Las etapas propuestas por Osbome (1991): preliminar, enfoque, confrontación y aplicación de acuerdo a lo planteado por el aprendizaje generativo.

> La historia de la electrización tomando como fundamento a Tales de Mileto y a William Gilbert quienes son los preaursores de la investigación en tomo a la electrización.

> Mileto con su vivencia sencilla con el Ámbar, experimento propuesto en la primera actividad.

$>$ Gilbert y su comprobación del fenómeno en otros materiales diferentes al Ámbar experimento siguiente

D Las ideas previas de los estudiantes como punto de partida de cada una de las actividades.

> Las competencias científicas argumentativa, interpretativa y propositiva que aparecen como parte integrante en cada una de las actividades experimentales. 


\section{Conclusiones}

La enseñanza de la física requiere para ir solventando cada uno de sus problemas la indusión de estrategias de e-a-e que permitan el aprendizaje significativo por parte de los estudiantes.

Con la implementación de la primera actividad se pudo conduir que:

> La historia de la ciencia además de servir como hilo conductor de las actividades experimentales fue un elemento dave para motivar a los estudiantes e introducirlos al desarrollo de cada experimento.

$>$ El análisis de la historia de la electrización permitió identificar elementos daves para el desarrollo de la propuesta, de los cuáles dos son considerados los más relevantes: primero el reconocimiento de las ideas que presentaron los cient́ficos en el momento de construcción del concepto, las auales al ser contrastadas con las ideas actuales de los estudiantes concuerdan; y segundo el hecho que de acuerdo a la historia, el conocimiento en tomo a un fenómeno ha sido construido partiendo de la actividad experimental.

> Las actividades planteadas según la metodología permitieron el desarrollo de competencias cientúficas en los estudiantes, especialmente las de tipo argumentativa, interpretativa y propositiva.

\section{Bibliografía}

Cubero, R. (1994). Concepciones alternativas, preconceptos, errores conceptuales. ¿Distinta terminología y un mismo significado? En Investigación en la Escuela, 23, pp. 33-35.

Driver R, Bell. (1986). Students' thinking and the leaming of saiencie: a constructivist view'. School Science Review, 67.

Colado J. (2003). Estructura Didáctica de las Actividades Experimentales de Ciencias Naturales para el Nivel Medio. [Tesis Doctoral]. La Habana, Cuba: Instituto Superior Pedagógico "Enrique José Varona".

Osbome R, freyberg P (1991). El aprendizaje de las ciencias: implicaciones en la ciencia de los alumnos. Narcea S.A de ediciones.

Quintanilla G. (2005). Identificación y caracterización de competencias científicas en el aula, ¿qué cambia en la enseñanza y en los nuevos modelos de conocimiento? Foro Nacional Educativo. 